23 HIDDEN: A HOSPICE INPATIENT DEEP VEIN THROMBOSIS DETECTION STUDY TO EXPLORE THE PREVALENCE, SYMPTOM BURDEN AND NATURAL HISTORY OF VENOUS THROMBOEMBOLISM IN PEOPLE WITH ADVANCED CANCER

MJ Johnson, SIR Noble, F Swan, V Allgar, E Napier, A Nelson, M Watson, C White. University of Hull, University of Cardiff, University of York, Belfast NHS Trust, Hospice UK, Northern Ireland Hospice

\subsection{6/bmjspcare-2019-ASP.22}

Background The deep venous thrombosis (DVT) prevalence in advanced cancer is unconfirmed and it is unknown whether current international thromboprophylaxis guidance is applicable to this group. We determined prevalence and predictors of femoral DVT in patients admitted to specialist palliative care units (SPCU).

Methods Prospective longitudinal observational study in five SPCUs in England, Wales and Northern Ireland. Consecutive adults with cancer underwent bilateral femoral vein ultrasonography on admission and weekly until death or discharger for a maximum of three weeks. Data were collected on performance status, attributable symptoms and variable known to be associated with venous thromboembolism. Patients were ineligible if admitted for terminal care (estimated prognosis < five days). Prevalence was estimated with 95\% confidence intervals (CI). DVT predictors and survival were explored using regression analyses. Sensitivity analysis excluded early scans to account for a technical learning curve.

Results 343 participants (68.2 [SD 12.8] 25 to 102 years; men 52\%; AKPS 49 [SD 16.6] 20\% to 90\%) were recruited. Of 273 evaluable scans, 92 (34\%, CI 28\% to 40\%) showed DVT. Excluding early scans, 64/232 (28\%, 22\% to 34\%) showed DVT. Four participants with a 'no DVT' scan on admission developed a DVT on repeat scanning over 21 days. Previous thromboembolism, bedbound $\leq 12$ weeks for any reason $(p=0.003)$ and lower limb oedema $(p=0.009)$ independently predicted DVT. Serum albumin $(p=0.430)$, thromboprophylaxis $(p=0.173)$ and survival $(p=0.473)$ were unrelated to DVT.

Conclusions These novel data show approximately one third of SPCU admissions with advanced cancer had a femoral DVT. DVT was not associated with thromboprophylaxis, survival or symptoms other than leg oedema. Findings are consistent with VTE being a manifestation of advanced disease rather than a cause of premature death. Thromboprophylaxis for SPCU inpatients with poor performance status seems of little benefit.

\section{EXPERIENCES OF OLDEST-OLD CARERS WHOSE PARTNER IS APPROACHING END-OF-LIFE: A SYSTEMATIC REVIEW AND NARRATIVE SYNTHESIS}

Tessa Morgan, Aamena Bharmal, Robbie Duschinsky, Stephen Barclay. University of Cambridge

\subsection{6/bmjspcare-2019-ASP.23}

Background Globally ageing populations have rapidly increased the number of people requiring palliative care. In resourcelimited healthcare systems, spouses are increasingly providing end-of-life care to enable their partners to remain at home. Little attention, however, has been paid to oldest-old spousal carers who are often managing their own co-morbidities whilst caring.
Aim To review the literature concerning experiences of carers aged 75 and over whose partner is approaching end-of-life.

Methods Systematic review and narrative synthesis of the empirical literature published between 1985-April 2018 across six databases. Gough's weight of evidence and Morgan's feminist appraisal tool used to determine quality of papers.

Results From the initial 6950 titles, eight qualitative studies and six quantitative studies were included. Five studies focused exclusively on care recipients with severe dementia. We identified three themes. (1) 'The embodied impact of care'. Many oldest-old carers experienced a 'double jeopardy' while providing emotionally and physically difficult end-of-life care and managing their own diminishing health. They often subordinated their own health needs to continue caring. (2) 'Marital and gendered conceptualisations of caring'. Oldest-old carers strove for normality in their self and marriage identities by providing care for their spouse with little additional support and by incorporating their caregiving within their established gender roles. (3) 'Learning to care'. Oldest-old carers had to learn new skills and ways of coping to keep their spouses at home. They became the expert of their partners' condition as well as having to take over all household tasks.

Conclusion This review highlights the importance of oldest-old carers contributions to their spouses' end-of-life care. Oldestold carers see it as their duty to care, but the caregiving itself involves learning new practical and emotional skills. Future research needs to consider how to best support these carers to do so without grave impact to their own health.

\section{Poster presentations}

\section{Bereavement | Posters 1 - 3}

\section{RETURNING TO WORK IN SPECIALIST PALLIATIVE CARE FOLLOWING PERSONAL BEREAVEMENT}

Paul Davies, Adam Hurlow, Suzie Gillon. Leeds Teaching Hospitals NHS Trust

\subsection{6/bmjspcare-2019-ASP.24}

Background During their working career, most people will experience a personal bereavement. Our perception was that a return to work might be difficult in a speciality with high patient mortality rates. Despite this, there is a paucity of evidence concerning the impact of bereavement on palliative care professionals. Staff recruitment and retention remains an ongoing challenge requiring optimisation of workforce support.

This study sought to elicit the factors that affect palliative care team-members' return to work following personal bereavement.

Methods An anonymised questionnaire based survey was distributed to specialist palliative care teams in Yorkshire for completion by clinical staff with patient contact.

Responses were analysed thematically.

Results 408 responses were returned from 857 questionnaires distributed (48\% response rate), of which 257 staff members had experienced personal bereavement whilst working in palliative care. Of the 169 who had absence from work: $35 \%$ were absent $\leq 1$ week; $46 \%>1$ week and $\leq 4$ weeks; $12 \%>4$ weeks $\leq 8$ weeks, and $6.5 \%>8$ weeks. A number of staff found returning to work challenging. There was significant variation in perceived support from colleagues; managers and organisations. 